\title{
BMJ Open Effectiveness of interventions to reduce COVID-19 transmission in a large urban jail: a model-based analysis
}

\author{
Giovanni S P Malloy (D) , ${ }^{1}$ Lisa Puglisi, ${ }^{2,3}$ Margaret L Brandeau, ${ }^{1}$ Tyler D Harvey, ${ }^{4}$ \\ Emily A Wang ${ }^{2,4}$
}

To cite: Malloy GSP,

Puglisi L, Brandeau ML, et al. Effectiveness of interventions to reduce COVID-19 transmission in a large urban jail: a modelbased analysis. BMJ Open 2021;11:e042898. doi:10.1136/ bmjopen-2020-042898

- Prepublication history for this paper is available online. To view these files, please visit the journal online (http://dx.doi org/10.1136/bmjopen-2020042898).

Received 18 July 2020 Revised 21 November 2020 Accepted 14 January 2021

Check for updates

(C) Author(s) (or their employer(s)) 2021. Re-use permitted under CC BY-NC. No commercial re-use. See rights and permissions. Published by BMJ.

${ }^{1}$ Management Science and Engineering, Stanford University, Stanford, California, USA

${ }^{2}$ Internal Medicine, Yale University, New Haven,

Connecticut, USA

${ }^{3}$ Pain Research, Informatics, Multimorbidities and Education Center, VA Connecticut Healthcare System, West Haven, USA

${ }^{4}$ SEICHE Center for Health and Justice, School of Medicine, Yale University, New Haven, Connecticut, USA

Correspondence to Mr Giovanni S P Malloy; malloyg@stanford.edu

\section{ABSTRACT}

Objectives We aim to estimate the impact of various mitigation strategies on COVID-19 transmission in a US jail beyond those offered in national guidelines.

Design We developed a stochastic dynamic transmission model of COVID-19.

Setting One anonymous large urban US jail.

Participants Several thousand staff and incarcerated individuals.

Interventions There were four intervention phases during the outbreak: the start of the outbreak, depopulation of the jail, increased proportion of people in single cells and asymptomatic testing. These interventions were implemented incrementally and in concert with one another.

Primary and secondary outcome measures The basic reproduction ratio, $R_{0}$, in each phase, as estimated using the next generation method. The fraction of new cases, hospitalisations and deaths averted by these interventions (along with the standard measures of sanitisation, masking and social distancing interventions).

Results For the first outbreak phase, the estimated $R_{0}$ was 8.44 (95\% credible interval (Crl): 5.00 to 13.10$)$, and for the subsequent phases, $R_{0 \text {, phase } 2}=3.64(95 \% \mathrm{Crl}$ : 2.43 to 5.11$), R_{0, \text { phase } 3}=1.72$ ( $95 \%$ Crl: 1.40 to 2.12 ) and $R_{0, \text { phase } 4}=0.58$ (95\% Crl: 0.43 to 0.75$)$. In total, the jail's interventions prevented approximately $83 \%$ of projected cases, hospitalisations and deaths over 83 days.

Conclusions Depopulation, single celling and asymptomatic testing within jails can be effective strategies to mitigate COVID-19 transmission in addition to standard public health measures. Decision makers should prioritise reductions in the jail population, single celling and testing asymptomatic populations as additional measures to manage COVID-19 within correctional settings.

\section{INTRODUCTION}

COVID-19, the disease caused by the SARS-CoV-2 virus, has affected millions of people worldwide, with disproportionate impact on some communities including those inside correctional facilities. In the USA, approximately 2.2 million people are incarcerated in any given day in over 5000 facilities, ${ }^{1}$ where the built environment and activities of daily living make physical

\section{Strengths and limitations of this study}

- COVID-19 has entered hundreds of correctional facilities in the USA, yet few empirical studies have focused on COVID-19 transmission in correctional facilities.

- We developed a stochastic dynamic transmission model describing the spread of COVID-19 in a large urban jail in the USA and calibrated the model to a moving average of the daily incident cases of COVID-19 reported by the jail.

- We identified three major interventions-depopulation, single celling and asymptomatic testing-undertaken by the jail and quantified the reduction in transmission rate as a result of these interventions.

- We report the estimated reduction in predicted cases, hospitalisations and deaths as a result of the jail interventions among both incarcerated people and correctional staff.

- The model assumes homogeneous mixing and does not capture transmission to and from the surrounding community.

distancing exceedingly difficult to implement. $^{2-4}$ As of the third week of April 2020, 420 US correctional facilities had at least one diagnosed case of COVID-19, accounting for a total of 4893 cases among incarcerated individuals and 2778 cases among staff members. ${ }^{3}$ As of June, correctional facilities accounted for 8 out of 10 of the largest COVID-19 outbreaks nationally, surpassing nursing homes and food processing plants, and 26 states had a higher rate of COVID-19 infection in their correctional population than in their general population. ${ }^{56}$ In spring 2020, Cook County Jail had one of the largest outbreaks in the country, and the infection rate at Rikers Island was nearly five times that of New York City. ${ }^{78}$

Despite the severity of outbreaks in correctional facilities, national guidance surrounding the prevention and management of COVID-19 within such settings has been limited. In the weeks after the first 
major outbreak in a US jail, the US Centers for Disease Control and Prevention (CDC) published policy guidelines for correctional facilities to help mitigate COVID-19 transmission; these included limiting transfer of incarcerated people between facilities, restricting the number of visitors entering facilities, promoting personal hygiene and environmental sanitisation, maximising the space between those incarcerated (ie, arranging bunks so individuals sleep head to toe) and screening staff for symptoms. ${ }^{9}$

However, CDC guidelines then and still now do not account for the difficulty that many facilities face in managing COVID-19. Even among those facilities which are not crowded, physical distancing is challenging given use of congregate living arrangements, shared meals, and exercise and recreation programming. In the absence of more targeted guidelines, there is wide variance in how correctional facilities are managing COVID-19, especially regarding depopulation efforts that may mitigate COVID-19 and approaches to testing (symptomatic only vs asymptomatic, viral testing vs antibody testing). As an example, Attorney General Barr has ordered that medically frail individuals in federal prisons be released to home quarantine, whereas many US state prison systems have no stated policies for larger scale release. Some correctional systems have implemented a onetime system-wide testing of all incarcerated individuals, including those who are asymptomatic, while others are only testing those who are symptomatic.

The effectiveness of various mitigation measures, many of which fall outside of CDC guidance, in reducing the transmission of COVID-19 within correctional facilities has yet to be established. In this study, we estimate the effectiveness of measures beyond standard CDC recommendations to mitigate the spread of COVID-19 in a large urban jail. With the aim of providing guidance to correctional policymakers and public health agencies, we focus on policies that could have large impact and are highly variable in implementation, namely depopulation (cessation of new detentions and release of incarcerated individuals), single celling (increasing the percentage of the total incarcerated population in a single cell) and testing asymptomatic individuals.

\section{METHODS}

We developed a stochastic dynamic transmission model of COVID-19 which we calibrated to the outbreak in the jail. We combined data on cases among incarcerated people and correctional staff because they interact very closely and regularly as an ecosystem behind the walls of the jail. Cases were confirmed using SARS-CoV-2 nasal swab PCR tests. We divided the outbreak timeline into four intervention phases marked by the start of the outbreak, start of depopulation efforts, increased single celling and large-scale asymptomatic testing of incarcerated individuals. We estimated the initial basic reproduction ratio, $R_{0}$, and the effective reproduction ratio, $R_{t}$, in each

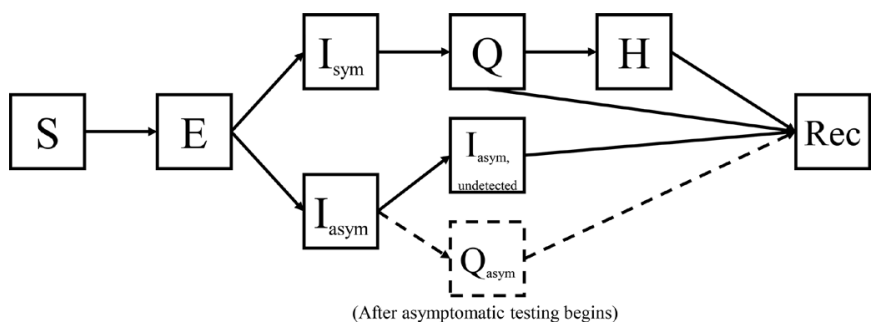

Figure 1 Structure of the disease transmission model. The disease states included susceptible $(S)$, exposed $(E)$, infected symptomatic $\left(I_{\text {sym }}\right)$, infected asymptomatic $\left(I_{\text {asym }}\right)$, infected asymptomatic undetected ( $\left.I_{\text {asym,undetected }}\right)$, quarantined $(Q)$, quarantined asymptomatic $\left(Q_{\text {asym }}\right)$, hospitalised $(H)$ and recovered $(R e c)$ individuals. Detection and subsequent quarantine of asymptomatic individuals are only considered after the start of asymptomatic testing in phase 4.

phase, for the entire jail. We also estimated the fraction of new cases, hospitalisations and deaths averted by the combined interventions in addition to the standard CDC recommended guidance.

\section{Model description}

We modified a traditional SEIR model to represent the disease states of COVID-19. These disease states included susceptible $(S)$, exposed $(E)$, infected symptomatic $\left(I_{s y m}\right)$, infected asymptomatic $\left(I_{a s y m}\right)$, infected asymptomatic undetected $\left(I_{\text {asym, undetected }}\right)$, quarantined symptomatic $(Q)$, quarantined asymptomatic $\left(Q_{\text {assm }}\right)$, hospitalised $(H)$ and recovered $(R e c)$ individuals (figure 1$)$. Individuals in the infected states $\left(I_{\text {symm }}, I_{\text {assm }}, I_{\text {assm, undetected }}\right)$ are assumed to be infectious, whereas individuals in the exposed state $(E)$ are not yet infectious. Some correctional systems distinguish between quarantining exposed groups together and isolating confirmed cases. In this model, the quarantined state includes both.

To model these interacting populations, we developed a mass-action mixing model described by the following equations:

$$
\begin{aligned}
& \frac{\mathrm{d} S}{\mathrm{dt}}=\mathrm{bS}-\frac{\beta \mathrm{S}}{\mathrm{N}}\left(\mathrm{I}_{\text {sym }}+\mathrm{I}_{\text {asym }}+\mathrm{I}_{\text {asym,undetected }}\right) \\
& \frac{\mathrm{dE}}{\mathrm{dt}}=\mathrm{bE}+\frac{\beta \mathrm{S}}{\mathrm{N}}\left(\mathrm{I}_{\text {sym }}+\mathrm{I}_{\text {asym }}+\mathrm{I}_{\text {asym,undetected }}\right)-\varepsilon \mathrm{E} \\
& \frac{\mathrm{dI}_{\mathrm{sym}}}{\mathrm{dt}}=(1-\alpha) \varepsilon \mathrm{E}-\mathrm{I}_{\mathrm{sym}} \\
& \frac{\mathrm{dI}_{\text {asym }}}{\mathrm{dt}}=\mathrm{bI}_{\text {asym }}+\alpha \varepsilon \mathrm{E}-\mathrm{I}_{\text {asym }} \\
& \frac{\mathrm{dI}_{\text {asym,undetect }}}{\mathrm{dt}}=\mathrm{bI}_{\text {asym,undetect }}+\left(1-\mathrm{p}_{\text {detected }}\right) \mathrm{I}_{\text {asym }}-\gamma \mathrm{I}_{\text {asym,undetect }} \\
& \frac{\mathrm{dQ}}{\mathrm{dt}}=\mathrm{I}_{\text {sym }}-(1-\eta) \gamma \mathrm{Q}-\eta\left(\frac{1}{\gamma}-\frac{1}{\mu}\right)^{-1} \mathrm{Q} \\
& \frac{\mathrm{d} Q_{\text {asym }}}{\mathrm{dt}}=\text { p }_{\text {detected }} \mathbf{I}_{\text {asym }}-\gamma \mathbf{Q}_{\text {ssym }} \\
& \frac{\mathrm{dH}}{\mathrm{dt}}=\eta\left(\frac{1}{\gamma}-\frac{1}{\mu}\right)^{-1} \mathrm{Q}-\mu \mathrm{H} \\
& \frac{\mathrm{dRec}}{\mathrm{dt}}=\left(1-\mathrm{d}_{\mathrm{I}}\right)(1-\eta) \gamma \mathrm{Q}+\left(1-\mathrm{d}_{\mathrm{I}}\right) \mu \mathrm{H}+\gamma \mathrm{I}_{\text {asym,undetect }}+\gamma \mathbf{Q}_{\mathrm{asym}} \\
& \frac{\mathrm{dDead}}{\mathrm{dt}}=\mathrm{d}_{\mathrm{I}}(1-\eta) \gamma \mathrm{Q}+\mathrm{d}_{\mathrm{I}} \mu \mathrm{H} \\
& \mathrm{N}=\mathrm{S}+\mathrm{E}+\mathrm{I}_{\text {sym }}+\mathrm{I}_{\text {asym }}+\mathrm{I}_{\text {asym,undetect }}+\mathrm{Q}+\mathrm{Q}_{\text {asym }}+\mathrm{H}+\mathrm{Rec}
\end{aligned}
$$

The susceptible, exposed and asymptomatic infected populations grew at rate $b$ which represented the overall growth or reduction in jail population. We assume that 
symptomatic infected individuals are not removed from the jail during general depopulation and would be admitted directly to quarantine. For the time horizon of the model, the population was generally shrinking. Susceptible individuals were exposed to COVID-19 at transmission rate $\beta$. We recalibrated this transmission rate for each of the four outbreak phases. We assumed that asymptomatic and symptomatic infected individuals could transmit the disease. ${ }^{10}{ }^{11}$ Exposed individuals were not yet infectious and become asymptomatic or symptomatic infected at rate $\varepsilon$, which corresponded to the incubation period of COVID-19. A certain proportion, $\alpha$, of these individuals stayed asymptomatic, while remaining individuals became symptomatic. Based on the jail's report, we assumed that symptomatic infected individuals and a fraction, $p_{\text {detected }}$, of asymptomatic infected individuals were identified 1 day after symptoms presented and placed in quarantine after identification. We assumed that individuals once quarantined did not transmit COVID-19, as they were isolated from the susceptible population. A fraction, $\eta$, of quarantined individuals were hospitalised and recovered from hospitalisation at rate $\mu$. All infected individuals recovered or died at rate $\gamma$ regardless of symptomatic or asymptomatic status. Symptomatic infected individuals died with probability $d_{r}$.

\section{Interventions}

The jail implemented various measures over time to mitigate the spread of COVID-19. We divide the outbreak into four intervention phases, corresponding to the initiation of key measures of interest which fell outside the guidance of the CDC. The interventions were implemented incrementally and in an additive manner, with depopulation first added, then single celling added, then asymptomatic testing added. The days when these interventions were added are shown in figure 2.

During phase 1 (days 1-11), the jail implemented a broad array of strategies that were consistent with CDC guidance including: basic screening for influenza-like symptoms in incarcerated people; new detainees quarantined for at least 7 days; basic screening for influenza-like symptoms for visitors, vendors, attorneys and community members entering the facility; staff required to report symptoms, as well as contact with known COVID-19 positive cases and any travel outside of the USA; suspension of all tours, large gatherings and in-person visitation. Sanitation techniques continued to follow CDC guidance for the duration of the outbreak and no significant new techniques were introduced during any other phases. A total of 23 SARS-CoV-2 tests were performed in this phase; 19 were positive (positivity rate $82.6 \%$ ).

During phase 2 (days 12-17), the jail population started to decrease by $1.41 \%$ each day through a combination of measures which included a marked decrease in new detentions given changes in court and judicial system procedures and large community organised bail outs (figure 2). The jail also began taking the temperature of all employees each day. Lastly, they started on-site voluntary

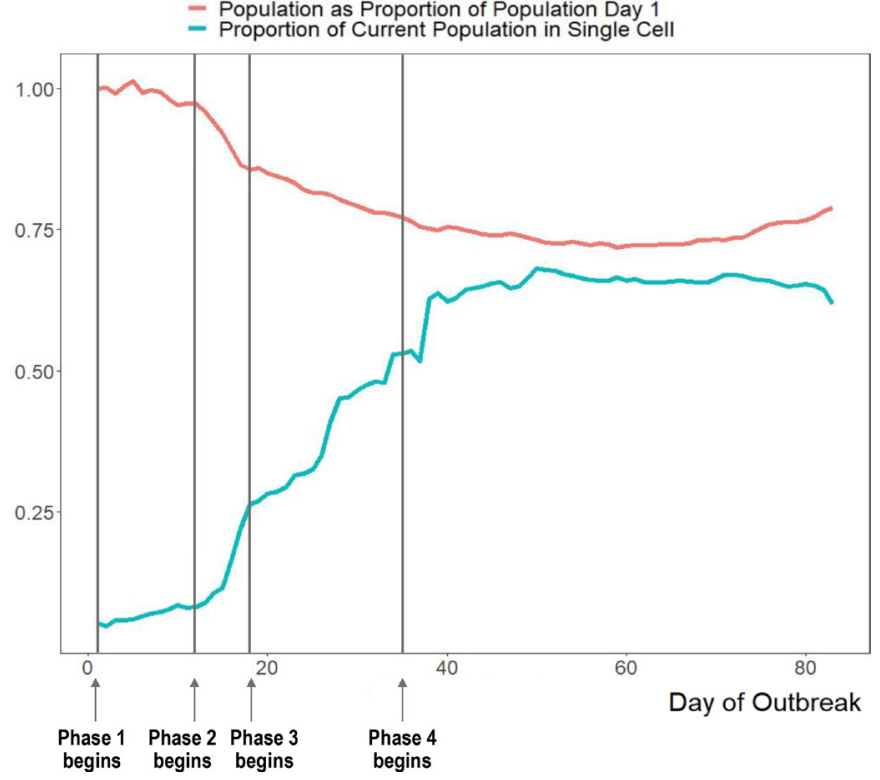

Figure 2 Change in the total population of the jail and the portion of the population in single-occupancy cells over the course of the outbreak. As depopulation increases, the overall population as a proportion of the population on day 1 of the outbreak decreases. Additionally, the proportion of incarcerated people in single-occupancy cells increases over time. We denote the timing of each intervention phase on the graph. Phase 1: initial outbreak, phase 2: depopulation began, phase 3 : increased single celling, phase 4 :

widespread testing of asymptomatic incarcerated individuals.

testing for employees and a 2-week COVID-19 paid leave policy for all employees. A total of 149 SARS-CoV-2 tests were performed in this phase; 139 were positive (positivity rate $93.2 \%$ ).

During phase 3 (days 18-36), the jail began increasing the proportion of the population in single-occupancy cells from $26 \%$ on day 18 to $54 \%$ on day 36 . During this period, they began requiring all staff to wear surgical masks and allotted new masks to those incarcerated each day. They also continued to isolate confirmed and suspected COVID-19 cases among incarcerated individuals. At this time, given the growing number of individuals, they identified a different building for segregating patients which provided a larger space for confirmed cases. A total of 455 SARS-CoV-2 tests were performed in this phase; 253 were positive (positivity rate $55.6 \%$ ).

During phase 4 (days 37-83), the jail began testing for asymptomatic cases at a rate of approximately 50-75 people per day in divisions with high numbers of cases identified during contact tracing. A total of 2741 SARS-CoV-2 tests were performed in this phase; 523 were positive (positivity rate $19.5 \%$ ).

\section{Model instantiation and calibration}

We estimated some model parameter values from previous literature (table 1). The rate at which exposed individuals became asymptomatically or symptomatically infected, $\varepsilon$, was the inverse of the incubation period. The incubation period of COVID-19 was previously described with a 


\begin{tabular}{|c|c|c|c|}
\hline Name & Description & Value & Source \\
\hline \multirow[t]{3}{*}{$b$} & Net rate of entrance into the jail, phase 1 (1/day) & -0.004 & \multirow{3}{*}{$\begin{array}{l}\text { Jail } \\
\text { dataset }\end{array}$} \\
\hline & Net rate of entrance into the jail, phase 3 (1/day) & -0.0076 & \\
\hline & Net rate of entrance into the jail, phase 4 (1/day) & 0.0005 & \\
\hline$\varepsilon$ & Incubation period ${ }^{-1}$ (1/day) & $\begin{array}{l}0.18 \\
\text { Incubation period: lognormal }(5.1, \\
0.89\end{array}$ & 12 \\
\hline$\alpha$ & Proportion of cases that are asymptomatic & $\begin{array}{l}0.405 \\
\text { uniform }(0.25-0.56)\end{array}$ & 1314 \\
\hline$\eta$ & Proportion of symptomatic infections that are hospitalised & 0.14 & $\begin{array}{l}\text { Jail } \\
\text { dataset }\end{array}$ \\
\hline$\mu$ & Recovery rate from hospital (1/day) & $\begin{array}{l}0.2 \\
\text { Length of hospitalisation: lognormal } \\
(5,1)\end{array}$ & 16 \\
\hline$d_{1}$ & Probability of death due to symptomatic COVID-19 infection & 0.01 & $\begin{array}{l}\text { Jail } \\
\text { dataset }\end{array}$ \\
\hline
\end{tabular}

lognormal distribution with mean 5.1 days and SD 0.89 days. ${ }^{12}$ We assumed that the proportion of infections that are asymptomatic, $\alpha$, was uniformly distributed over the range $0.25-0.56 .^{1314}$ The average recovery rate was previously estimated to be 0.1 , the inverse of the 10-day mean infection period..$^{15}$ We assumed that the infection period followed a truncated normal distribution with mean 10 days, SD 6.25 days, minimum 5 days and maximum 20 days. Additionally, the average length of hospitalisation from COVID-19 has been estimated to be 5 days, making the daily recovery probability from the hospital 0.2. ${ }^{16}$ We assumed that the length of hospitalisation followed a lognormal distribution with a mean of 5 days and SD of 1 day.

The jail provided demographic data about the size of the incarcerated population per day, as well as epidemiological data about confirmed COVID-19 cases over the course of 83 days. We assumed an average reporting delay of 6 days from first exposure to reported incident cases. This accounts for the mean incubation period and a minor delay between symptom onset and isolation. The jail provided data on the age of the infected person, date of positive COVID-19 test, the work or incarceration location of the infected individual, and whether the individual was hospitalised or died as a result of the COVID-19 infection. Testing was performed on admission to the jail and through symptom onset or contact tracing. We used these data to calculate the proportion of symptomatic infections that were hospitalised or died. For each intervention phase, we used the epidemiological data to determine the growth rate, $b$, as the average rate of growth for the entire facility.

We calibrated the transmission rate, $\beta$, for each intervention phase. We first pseudo-randomly selected values for parameters $\varepsilon, \alpha, \gamma$ and $\mu$ based on our assumed distributions (table 1 ). Then, we calculated $b$ for the intervention phase. To find the best-fitting value of $\beta$ for the given parameter set, we implemented an exhaustive search over the range (0-4) in increments of 0.01 . We chose the value of $\beta$ which minimised the sum of mean squared error between the reported daily incident cases of confirmed COVID-19 cases among incarcerated people and staff in the jail to the daily incident cases of symptomatic infected cases in the model for that phase. We calculated incident symptomatic cases using the raw reported incident cases before asymptomatic testing. Select asymptomatic testing for incarcerated people began on day 31 and for staff began on site on day 21. After asymptomatic testing began, we took the minimum of the jail-provided data on the number of symptomatic tests multiplied by the average percentage of positive results of symptomatic tests between days 16-30 (89\%) and the raw reported incident cases. Based on this estimate, on average, $82 \%$ of the reported daily incident cases among the incarcerated population was symptomatic after asymptomatic testing began. Because we did not have testing data for staff, we assumed that $82 \%$ of reported new staff cases were symptomatic after on-site testing became available for staff.

We used a simple moving average of the previous 5 days of symptomatic incident cases to smooth the calibration 
targets. We assumed that the reported incident cases corresponded to the number of incarcerated individuals and staff members who showed symptoms of COVID-19. For each intervention phase, we ran 1000 Monte Carlo simulations and defined the $95 \%$ credible interval (CrI) of $\beta$ as the range in which $95 \%$ of calibrated values of $\beta$ fell.

\section{Calculation of $R 0$ and $R t$}

To calculate $R_{0}$ and $R_{t}$, we used the next generation method. ${ }^{17}$ This method uses two matrices of partial derivatives of compartments with infected individuals. ${ }^{18}$ In our model, this included exposed, asymptomatic infected, symptomatic infected, quarantined and hospitalised individuals. The first matrix, $F$, is the rate of appearance of new infections for each compartment. Each element, $f_{i j}$, of $F$ is the partial derivative of any term in which new infections appear in compartment $i$ with respect to compartment $j$ where $i, j \in\left[E, I_{\text {sym }}, I_{\text {asym, undelected }}\right.$, $\left.Q, Q_{a s y m}, H\right]$.

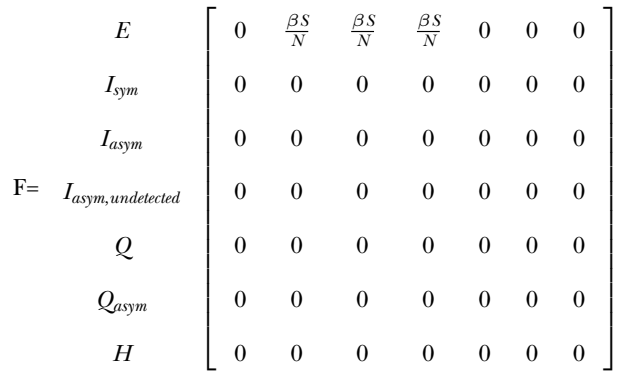

The second matrix, $V$, is the rate of transfer of individuals out of a compartment minus the rate of transfer of individuals into a compartment. Therefore, each element, $v_{i j}$, of $V$ is the partial derivative of the additive inverse of any term other than the appearance of new infections in compartment $i$ with respect to compartment $j$. The matrix $V$ and its inverse are as follows:

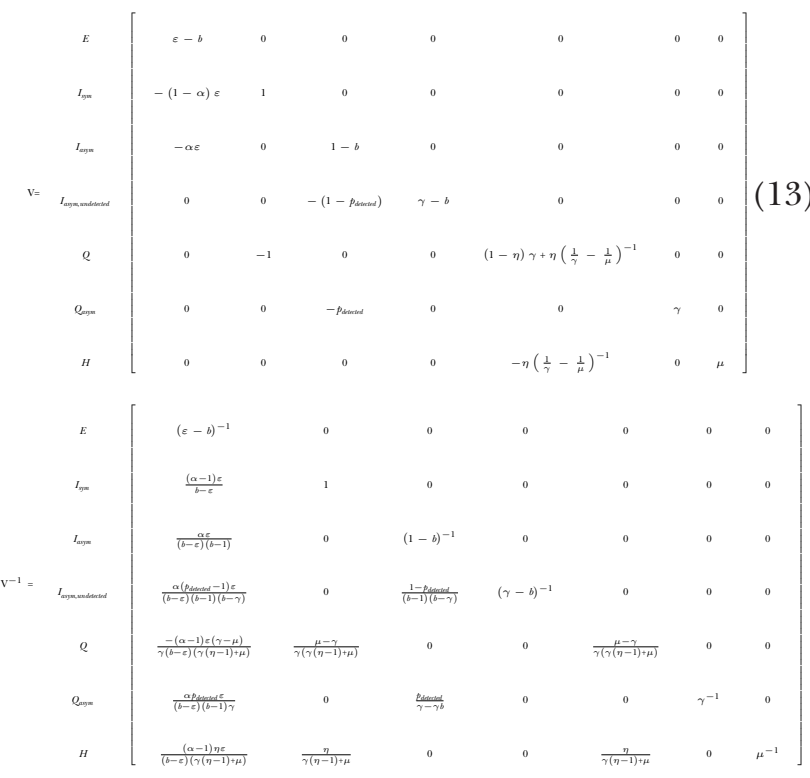

(14)
The next generation method calculates $R_{0}$ as the dominant eigenvalue of the next generation matrix. The next generation matrix is defined as $F V^{1}$ :
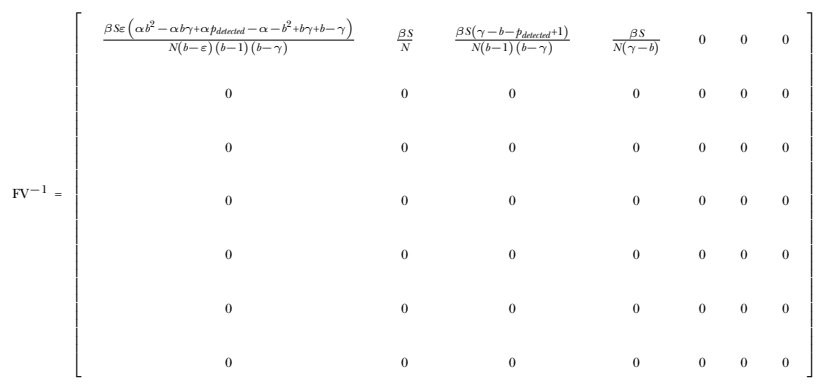

In our model, $F V^{-1}$ has only one non-zero eigenvalue, $\lambda=\frac{\beta S \varepsilon\left(\alpha \mathrm{b}^{2}-\alpha \mathrm{b} \gamma+\alpha \text { pdetected }^{2}-\alpha-\mathrm{b}^{2}+\mathrm{b} \gamma+\mathrm{b}-\gamma\right)}{\mathrm{N}(\mathrm{b}-\varepsilon)(\mathrm{b}-1)(\mathrm{b}-\gamma)}$. Therefore, $R_{0}=\max (0$, $\lambda$ ), and since $\lambda \geq 0, R_{0}=\lambda$. Since $R_{0}$ is directly proportional to $\beta$, we can calculate the values of $R_{0}$ of other phases simply by using phase 1 starting conditions combined with the reduced transmission rate.

To find the effective reproduction ratio, $R_{t}$, at time $t$, we used the next generation method with the same matrices but updated the values of $S$ and $\beta$ as appropriate. Because the number of susceptible individuals, $S$, is a function of time, we recalculate $R_{t}$ each day. The functional form of $R_{t}$ for our model is as follows:

$$
\mathrm{R}_{\mathrm{t}}=\frac{\beta \mathrm{S}_{\mathrm{t}} \varepsilon\left(\alpha \mathrm{b}^{2}-\alpha \mathrm{b} \gamma+\alpha \text { petected }-\alpha-\mathrm{b}^{2}+\mathrm{b} \gamma+\mathrm{b}-\gamma\right)}{\mathrm{N}(\mathrm{b}-\varepsilon)(\mathrm{b}-1)(\mathrm{b}-\gamma)}
$$

We computed the $95 \% \mathrm{CrI}$ of $R_{t}$ as the range in which $95 \%$ of calibrated values of $R_{t}$ fell.

\section{Sensitivity analysis}

We assumed that the average length of time in the exposed state (ie, the incubation period) was 5.1 days based on a study of 181 cases in Wuhan, China. ${ }^{12}$ Two recent studies estimated that $44 \%-48 \%$ of transmission can come from presymptomatic individuals, suggesting that the mean length of time in the exposed state could be shorter than we assumed. ${ }^{1920}$ Given this recent evidence, we performed sensitivity analysis where we reduced the mean length of time in the exposed state by 2.1 days, and correspondingly increased the mean length of time in the infectious state by 2.1 days. The mean value of $\varepsilon$ was updated accordingly to $\frac{1}{3}$. The model otherwise remained unchanged.

\section{Role of the funding source}

The funding sources had no role in the study design, collection, analysis and interpretation of data, writing the report nor the decision to submit the paper for publication.

Patient and public involvement

No patients were involved.

\section{RESULTS}

The number of daily reported incident cases of COVID-19 in the jail was highly variable, ranging from 0 to 67 . The 


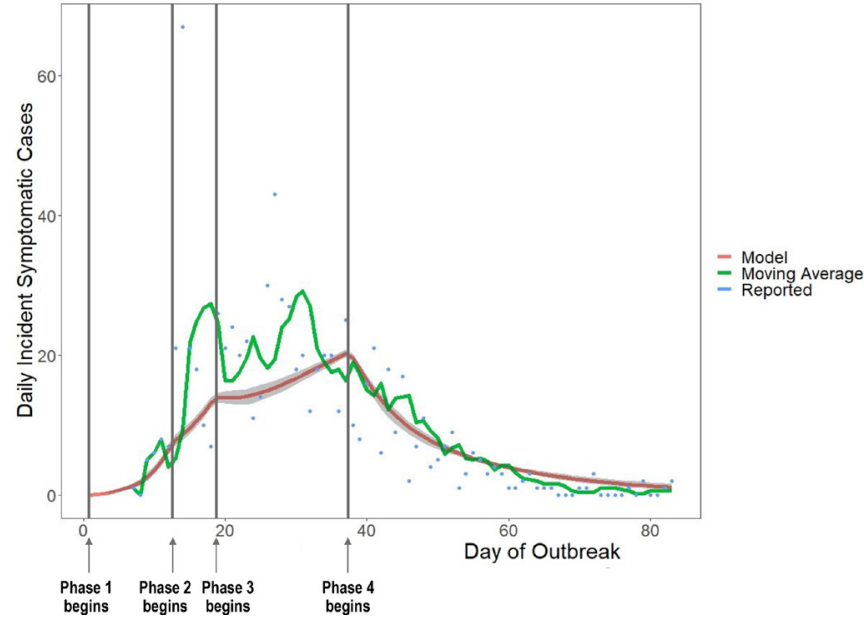

Figure 3 Comparison of the daily number of incident symptomatic cases in the model with reported new symptomatic COVID-19 cases at the jail. Shaded grey area represents $95 \%$ credible interval of model runs. We denote the timing of each intervention phase on the graph. Phase 1: initial outbreak, phase 2: depopulation began, phase 3: increased single celling, phase 4: widespread testing of asymptomatic incarcerated individuals.

mean absolute error of the model values compared with the simple moving average was $29 \%$ (figure 3 ).

\section{Transmission rates}

When following the initial CDC recommendations for correctional facilities (phase 1), the baseline transmission rate $(\beta)$ was 1.79 (95\% CrI: 1.35 to 2.22 ) (figure 4 ). After depopulation began (phase 2), the transmission rate was $\beta=0.78$ (95\% CrI: 0.61 to 0.97 ). This represents a $56 \%$ decrease in the transmission rate from phase 1. After the increase in single-occupancy cells (phase 3), the transmission rate was $\beta=0.38$ (95\% CrI: 0.28 to 0.52$)$, a

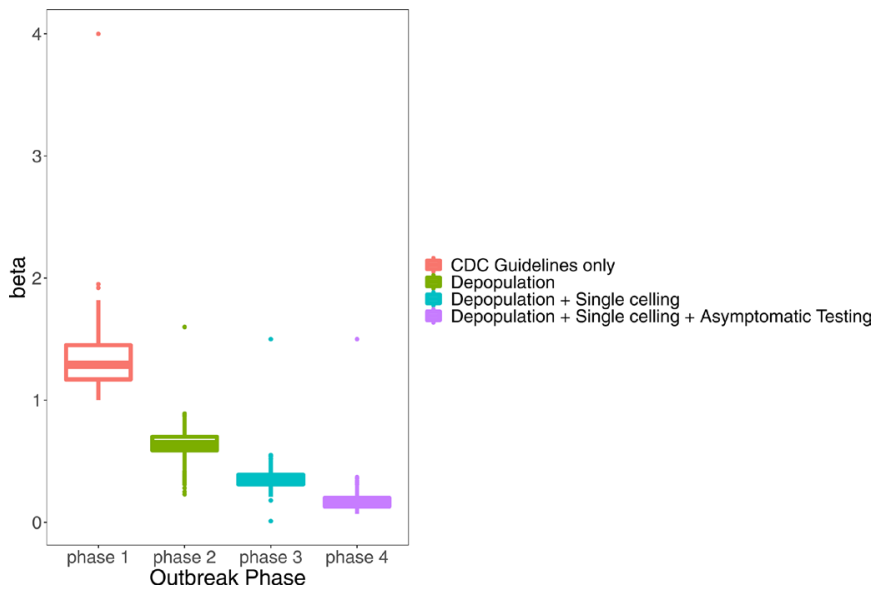

Figure 4 Calibrated values of the transmission rate $\beta$ for different outbreak phases (phase 1: initial outbreak, phase 2: depopulation began, phase 3: increased single celling, phase 4: widespread testing of asymptomatic incarcerated individuals). CDC guidelines were implemented during all four phases. Boxes denote 25th percentile, median and 75th percentile. CDC, Centers for Disease Control and Prevention.
$51 \%$ decrease from phase 2 . Finally, the transmission rate after testing of asymptomatic individuals began (phase 4) was $\beta=0.13$ (95\% CrI: 0.07 to 0.24 ), a $66 \%$ decrease from phase 3 . All of these reductions are statistically significant.

\section{Reproduction ratios}

The estimated value of $R_{0}$ was highest in phase 1 , during the first 11 days of the outbreak (table 2). For this phase, we estimate $R_{0}=8.44$ (95\% CrI: 5.00 to 13.10) (table 2). We estimate $R_{0}$ of each phase in a completely susceptible population as if the outbreak had begun with the values of $\beta$ which correspond to each phase: $R_{0 \text {, phase } 2}=3.64(95 \%$ CrI: 2.43 to 5.11 ), $R_{0, \text { phase } 3}=1.72$ (95\% CrI: 1.40 to 2.12 ) and $R_{0, \text { phase } 4}=0.58$ (95\% CrI: 0.43 to 0.75$)$. Figure 5 shows the effective reproduction ratio, $R_{t}$, over time for all intervention phases. $R_{t}$ decreased as the susceptible population shrank, the transmission rate changed and different interventions were implemented. For the entire jail, we estimate that the interventions may have reduced the effective reproduction ratio $R$ below 1 about 5 weeks after the outbreak began (on day 37 ).

\section{Averted infections, hospitalisations and deaths}

Table 2 shows the expected total symptomatic cases on day 83 and expected total cases on day 200, assuming that the estimated transmission rate for a particular outbreak phase holds over all subsequent days. Over the first 83 days of the outbreak, the jail reported 778 symptomatic cases, 67 hospitalisations and 10 deaths among incarcerated individuals and staff. Our model predicts 635 symptomatic cases (95\% CrI: 506 to 821$)$, 89 hospitalisations (95\% CrI: 71 to 115 ) and 6 deaths (95\% CrI: 5.8 to 6.7 ) over this same time period (figure 6 ). Our estimate is $18 \%$ less than the number of reported cases that were symptomatic. Compared with what could have happened with only the implemented CDC-recommended interventions of phase 1 , the model predicts a reduction of approximately 3100 symptomatic cases, 435 hospitalisations and 30 deaths over 83 days. This suggests that the combination of interventions (depopulation, increased single celling and large-scale asymptomatic testing of incarcerated individuals) in addition to standard CDC COVID-19 mitigation strategies led to an $83 \%$ reduction in predicted symptomatic cases, hospitalisations and deaths.

\section{Sensitivity analysis}

In sensitivity analysis, when we assumed an incubation period that was 2.1 days shorter, the calibrated baseline transmission rate was $\beta=1.31$ (95\% CrI: 1.00 to 1.71$)$. After depopulation began (phase 2), the transmission rate was $\beta=0.64$ (95\% CrI: 0.41 to 0.83 ). This represents a $51 \%$ decrease in the transmission rate from phase 1 (compared with a $56 \%$ decrease in the base case results). After the increase in single-occupancy cells (phase 3), the transmission rate was $\beta=0.36$ (95\% CrI: 0.25 to 0.49 ), a $44 \%$ decrease from phase 2 (compared with a $51 \%$ decrease in the base case results). Finally, the transmission rate after testing of asymptomatic individuals began (phase 


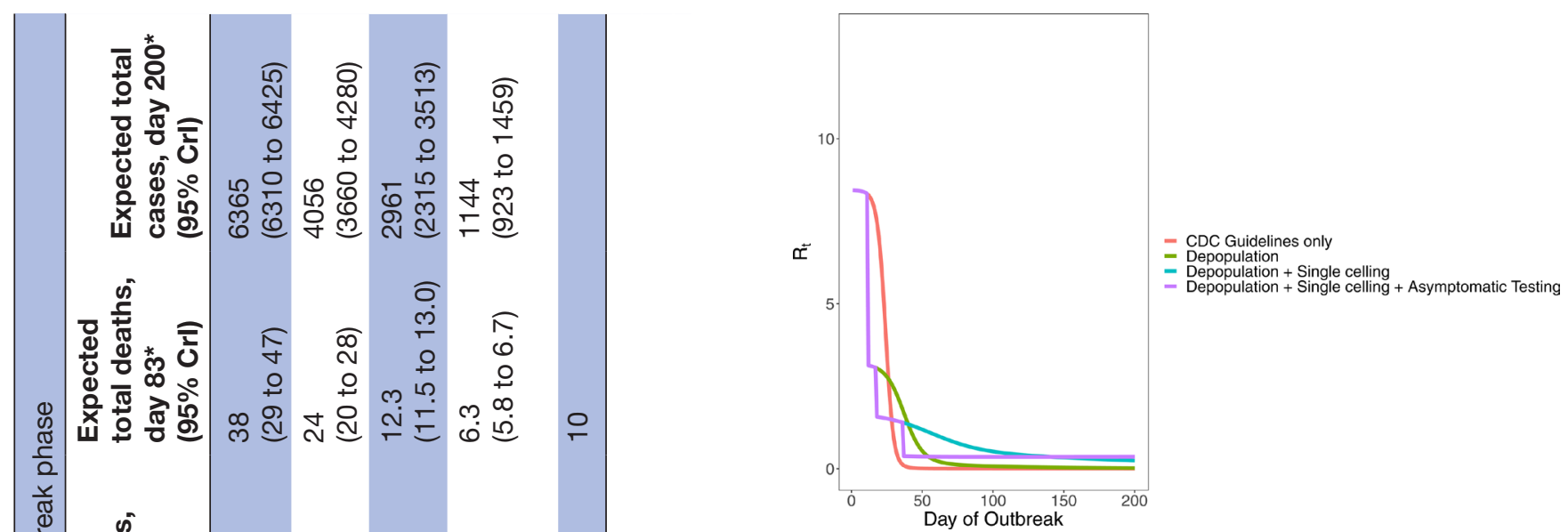

Figure 5 Calculated values of the effective reproduction ratio $R_{t}$ for all intervention phases (phase 1: initial outbreak, phase 2: depopulation began, phase 3: increased single celling, phase 4: widespread testing of asymptomatic incarcerated individuals). CDC guidelines were implemented during all four phases. Shaded area around each line reflects the $95 \%$ credible interval. CDC, Centers for Disease Control and Prevention.

4) was $\beta=0.17$ (95\% CrI: 0.09 to 0.30 ), a $53 \%$ decrease from phase 3 (compared with $66 \%$ in the base case). We estimate the following basic reproduction ratios: $R_{0}=6.22$ (95\% CrI: 3.56 to 9.98$), R_{0, \text { phase } 2}=3.02(95 \%$ CrI: 1.95 to $4.32), R_{0, \text { phase } 3}=1.64$ (95\% CrI: 1.33 to 2.02$)$ and $R_{0, \text { phase } 4}$ $=0.75$ (95\% CrI: 0.59 to 0.92$)$.

Over the first 83 days of the outbreak, the sensitivity analysis predicts 637 symptomatic cases (95\% CrI: 502 to 827 ), 89 hospitalisations (95\% CrI: 70 to 116 ) and 6 deaths (95\% CrI: 5.8 to 6.8 ), values very close to those predicted in the base case analysis. Thus, even assuming a shorter incubation period, we estimate that the mitigation

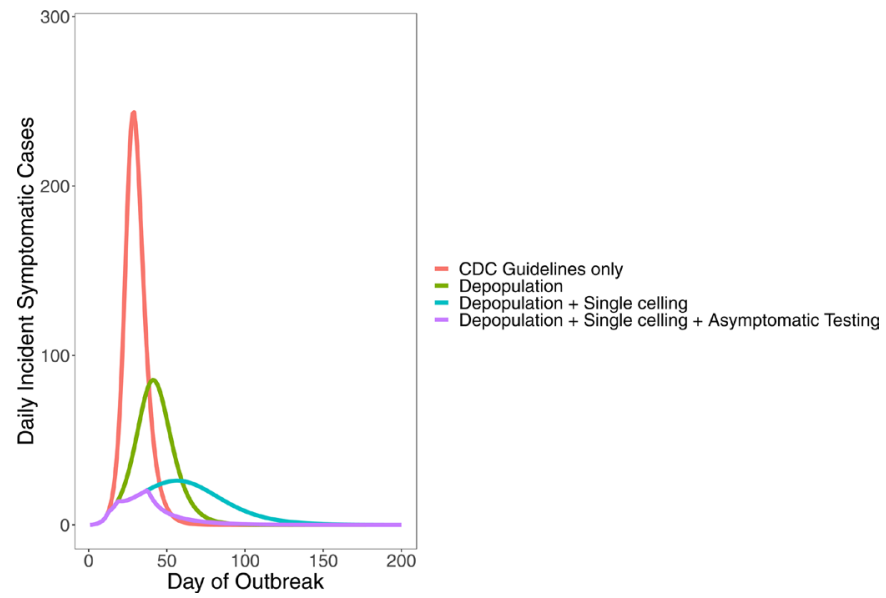

Figure 6 Projected number of incident symptomatic cases per day for all intervention phases (phase 1: initial outbreak, phase 2: depopulation began, phase 3: increased single celling, phase 4: widespread testing of asymptomatic incarcerated individuals). CDC guidelines were implemented during all four phases. Shaded area around each line reflects the $95 \%$ credible interval. CDC, Centers for Disease Control and Prevention. 
strategies led to an $83 \%$ reduction in predicted symptomatic cases, hospitalisations and deaths.

\section{DISCUSSION \\ Principal findings}

Using a stochastic compartmental model, we estimate that depopulation efforts, single celling and asymptomatic testing are important interventions to reduce COVID-19 transmission in jails. We estimate that these actions taken by the jail, in addition to those recommended by the CDC including sanitation and masking, reduced potential new cases by approximately $83 \%$ over 83 days, and this may have averted more than 435 hospitalisations and 30 deaths among those who live and work in the jail. Taken together, these measures not only have bearing for the correctional facility, but also for the community health systems that surround the jail.

\section{Policy implications}

Our findings suggest that depopulation efforts should be a primary strategy for COVID-19 mitigation in jails. Reduction in detained populations to prevent disease transmission is best achieved by both decreasing the number of new intakes and increasing the number of releases.

This requires that authorities controlling jail admissions (including police departments, judges and in some cases correctional departments) and jail releases (including judges, lawyers and community bail funds) focus on promoting depopulation efforts to mitigate COVID-19 transmission.

By creating smaller populations within correctional institutions, other mitigation strategies, including physical distancing and the ability to quarantine and medically isolate the incarcerated population that remains when necessary, are easier to implement. Our analysis suggests that single celling, in concert with depopulation, was effective in mitigating COVID-19 transmission. To be clear, single celling does not imply solitary confinement but rather placing one person in a $6 \times 9 \mathrm{ft}$ cell to increase physical distancing in correctional facilities. ${ }^{21}$ Given physical crowding in many facilities, even when overall incarcerated populations are at record lows, increasing access to single-occupancy cells will not be feasible without depopulation efforts, and as supported by our model, will not lead to a contained transmission rate alone. Depopulation should continue in concert with single celling, as depopulation reduces density of shared spaces in common areas. Facilities unable to appropriately place individuals in single cells without relying on solitary confinement should embrace depopulation as a preferred strategy. Decarceration will require interagency coordination to achieve the full public health impact, including testing people prior to release. ${ }^{22}$ Without testing and ensuring opportunities for community quarantine, correctional facilities may contribute to ongoing transmission in the surrounding community. ${ }^{23} 24$
Lastly, asymptomatic testing is an important component of COVID-19 mitigation strategies. This jail focused on asymptomatic testing through contact tracing of people who tested positive, medically vulnerable populations and on admission. However, more research needs to be conducted on who should be tested and under what circumstances, including whether mass testing is effective, when individuals should be tested and at what intervals.

National and international health agencies, such as the CDC and the WHO, should address depopulation, single celling and asymptomatic testing in future guidance for detention facilities and should consider how to best implement these measures. Correctional facility administrators will need to consider how to best mitigate the challenges that come with these strategies. For example, coordination of healthcare and social services organisations prior to release should be prioritised, as should considerations of testing when releasing individuals as part of depopulation efforts. ${ }^{42}$

\section{Limitations}

Our analysis has several limitations. We used a compartmental model which assumes homogeneous mixing among the entire population. Correctional facilities in reality do not exhibit homogeneous mixing, especially across divisions, buildings or tiers within the facility. Our model does not have the granularity to capture the influence of individuals on transmission dynamics. Our model assumes a relatively stationary population and only accounts for mixing within the jail. Jail populations are highly variable with frequent intakes and releases. Jailed individuals also have variable daily routines, such as where they eat or exercise, which are not accounted for in our model. We did not account for possible false positives, misdiagnosis, over-reporting or under-reporting in the dataset. Finally, the many interventions undertaken by the jail make it difficult to determine the causal influence of any one intervention.

Importantly, these limitations influence our estimates of $\beta$ and $R_{0}$. We model the jail as a closed system and thus neglect exogeneous infection (eg, staff or new intake incarcerated individuals who contracted the disease in the community) that likely entered the jail before largescale testing efforts. Because our analysis assumed that all new infections arise from internal transmission, we likely overestimate the true values of $\beta$ and $R_{0}$, particularly in the early phases of the epidemic in the jail. Thus, conclusions resulting from our analysis should focus on the relative reductions of $\beta$ and $R_{0}$ rather than the precise estimates of these values.

\section{CONCLUSIONS}

Despite the limitations of our analysis, we conclude that it is possible to mitigate the spread of COVID-19 even in correctional settings, where standard physical distancing practices are difficult to achieve, by implementing depopulation strategies, promoting increased single celling 
and asymptomatic testing with appropriate isolation. The large estimated reduction in the transmission rate $(\geq 80 \%)$ from these three intervention strategies is comparable with standard social distancing measures in a community setting. ${ }^{25}$ Even when accounting for potential additional presymptomatic transmission, the relative reductions in $\beta$ and $R_{0}$ remain very high, further reinforcing the effectiveness of depopulation, single celling and asymptomatic testing. As states and the federal government are focused on reopening economies and resurging numbers of cases in many states, strategies should be devised to protect those who are incarcerated and those who work in corrections by further limiting population increases so that future outbreaks are averted.

\section{Twitter Emily A Wang @ewang422}

Acknowledgements We acknowledge the anonymous people from the jail for their prompt collaboration and transparency in providing detailed data on COVID-19 transmission.

Contributors GSPM contributed to model design, creation and implementation, data analysis and manipulation, analysis of results, figure creation, and writing. LP contributed to writing, editing, data collection, study design and literature review. MLB contributed to model design, writing and editing. TDH contributed to writing, editing and literature search. EAW contributed to writing, editing, data collection, study design and literature search.

Funding At the time that this work was conducted, GSPM was supported by grant number T32HS026128 from the Agency for Healthcare Research and Quality, MLB was supported by grant R37-DA15612 from the National Institute on Drug Abuse and LP was partially supported by the Veterans Health Administration (grant number N/A). In the past 36 months, EAW received research support through Yale University from the Bureau of Justice Administration to study re-entry by linking correctional and community health system data (2015-RY-BX-K002) and the Substance Abuse and Mental Health Services Administration to study how to improve the health of women just released from corrections. EAW currently receives research support through Yale University from the National Cancer Institute of National Institute of Health (1R01CA230444), the National Heart, Lung and Blood Institute (1R01HL137696), the National Institute of Minority Health and Disparities (1R01MD010403), the National Institute of Drug Abuse (1UG1DA050072 and 3 UG1 DA050072-02S3), and the National Library of Medicine (1 R01LM013477-01) to study incarceration and cancer, cardiovascular disease, gun violence, opioid use disorder and COVID-19. She also receives funding from the William T Grant Foundation (188795) to study health-related barriers and facilitators to reducing criminal legal contact and from the California Healthcare Foundation (19-0 02 900) to evaluate the Transitions Clinic Network in California. GM, LP, MB, TH, and EW were supported by grant 3 UG1 DA050072-02S3 from the National Institute on Drug Abuse.

Disclaimer The content is solely the responsibility of the authors and does not necessarily represent the policy or views of any of the funding agencies.

Competing interests None declared.

Patient consent for publication Not required.

Ethics approval This study was deemed exempt from Institutional Review Board review by the Yale Human Investigation Committee as we received completely anonymised data from the jail.

Provenance and peer review Not commissioned; externally peer reviewed.

Data availability statement Data are available upon reasonable request. Whenever possible, in accordance with previously signed data usage agreements, we will make the data used in this study available upon reasonable request to GSPM.

Open access This is an open access article distributed in accordance with the Creative Commons Attribution Non Commercial (CC BY-NC 4.0) license, which permits others to distribute, remix, adapt, build upon this work non-commercially, and license their derivative works on different terms, provided the original work is properly cited, appropriate credit is given, any changes made indicated, and the use is non-commercial. See: http://creativecommons.org/licenses/by-nc/4.0/.
ORCID iD

Giovanni S P Malloy http://orcid.org/0000-0002-0806-5525

\section{REFERENCES}

1 Kaeble D, Cowhig M. Correctional populations in the United States. Washington, DC: U.S. Department of Justice, Bureau of Justice Statistics, 2018.

2 Hawks L, Woolhandler S, McCormick D. COVID-19 in prisons and jails in the United States. JAMA Intern Med 2020;180:1041-2.

3 Wallace M, Hagan L, Curran KG, et al. COVID-19 in Correctional and Detention Facilities - United States, February-April 2020. MMWR Morb Mortal Wkly Rep 2020;69:587-90.

4 Kinner SA, Young JT, Snow K, et al. Prisons and custodial settings are part of a comprehensive response to COVID-19. Lancet Public Health 2020;5:e188-9.

5 McCauley E. COVID case watch June 3, 2020. Available: https:// covidprisonproject.com/blog/ [Accessed 1 Aug 2020].

6 The New York Times. Coronavirus in the U.S: latest map and case count. New York Times, 2020.

7 Williams T, Ivory D. Coronavirus behind bars: Cook County jail is top U.S. hot spot. New York Times, 2020.

8 The Legal Aid Society. COVID-19 infection tracking in NYC jails. Available: https://legalaidnyc.org/covid-19-infection-tracking-in-nycjails/ [Accessed 24 Apr 2020].

9 Centers for Disease Control and Prevention. Interim guidance on management of coronavirus disease 2019 (COVID-19). Available: https://www.cdc.gov/coronavirus/2019-ncov/downloads/guidancecorrectional-detention.pdf [Accessed 1 Jun 2020].

10 Wei WE, Li Z, Chiew CJ. Presymptomatic transmission of SARSCoV-2 - Singapore. MMWR Morb Mortal Wkly Rep 2020;69:411-5.

11 Bai Y, Yao L, Wei T, et al. Presumed asymptomatic carrier transmission of COVID-19. JAMA 2020;323:1406-7.

12 Lauer SA, Grantz KH, Bi Q, et al. The incubation period of coronavirus disease 2019 (COVID-19) from publicly reported confirmed cases: estimation and application. Ann Intern Med 2020;172:577-82.

13 Oran DP, Topol EJ. Prevalence of Asymptomatic SARS-CoV-2 Infection : A Narrative Review. Ann Intern Med 2020;173:362-7.

14 Arons MM, Hatfield KM, Reddy SC, et al. Presymptomatic SARSCoV-2 infections and transmission in a skilled nursing facility. $N$ Engl J Med 2020;382:2081-90.

15 Rocklöv J, Sjödin H, Wilder-Smith A. COVID-19 outbreak on the diamond Princess cruise SHIP: estimating the epidemic potential and effectiveness of public health countermeasures. J Travel Med 2020;27:taaa030.

16 Thevarajan I, Nguyen THO, Koutsakos M, et al. Breadth of concomitant immune responses prior to patient recovery: a case report of non-severe COVID-19. Nat Med 2020;26:453-5.

17 Diekmann O, Heesterbeek JA, Metz JA. On the definition and the computation of the basic reproduction ratio $\mathrm{RO}$ in models for infectious diseases in heterogeneous populations. J Math Biol 1990;28:365-82.

18 Heffernan JM, Smith RJ, Wahl LM. Perspectives on the basic reproductive ratio. J $R$ Soc Interface 2005;2:281-93.

19 He X, Lau EHY, Wu P, et al. Temporal dynamics in viral shedding and transmissibility of COVID-19. Nat Med 2020;26:672-5.

20 Moghadas SM, Fitzpatrick MC, Sah P, et al. The implications of silent transmission for the control of COVID-19 outbreaks. Proc Natl Acad Sci U S A 2020;117:17513-5.

21 Cloud D, Augustine D, Ahalt C. The Ethical Use of Medical Isolation - Not Solitary Confinement - to Reduce COVID-19 Transmission in Correctional Settings. Available: https://amend.us/wp-content/ uploads/2020/04/Medical-Isolation-vs-Solitary_Amend.pdf [Accessed 1 Sep 2020].

22 National Academies of Sciences, Engineering and Medicine. Decarcerating correctional facilities during COVID-19: advancing health, equity, and safety. Washington DC, 2020.

23 Reinhart E, Chen DL. Incarceration and its disseminations: COVID-19 pandemic lessons from Chicago's Cook County jail. Health Aff 2020;39:1412-8.

24 Lofgren E, Lum K, Horowitz A. The epidemiological implications of incarceration dynamics in jails for community, corrections officer, and incarcerated population risks from COVID-19. Available: https:// www.medrxiv.org/content/10.1101/2020.04.08.20058842v2.full.pdf [Accessed 1 Jun 2020].

25 Lewnard JA, Lo NC. Scientific and ethical basis for social-distancing interventions against COVID-19. Lancet Infect Dis 2020;20:631-3. 